\title{
Angioplastia Coronária nas Indicações Off-Label: Comparação das Vias Radial vs. Femoral
}

\author{
Clarissa Campo Dall'Orto', Guilherme Alves Lapa', Joaquim Davi Carneiro Neto', \\ Nádia de Mendonça Carnieto', João Batista do Oliveira Neto1', Maria Fernanda Zuliani Mauro', \\ Salvador André Bavaresco Cristóvãoํㅜ, Adnan Ali Salman¹, José Armando Mangione ${ }^{1}$
}

\section{RESUMO}

Introdução: A via radial é objeto de interesse crescente para procedimentos diagnósticos e terapêuticos por possuir diversas vantagens, entre as quais comodidade para o paciente no pós-procedimento imediato, com retorno precoce a suas atividades, diminuição do tempo de internação, com consequente redução dos custos hospitalares, e baixo índice de complicação do sítio de punção comparativamente à via femoral, reduzindo a taxa de sangramento maior, que, por sua vez, está relacionada ao aumento do risco de morte e eventos isquêmicos. Método: Análise retrospectiva de 1.807 pacientes consecutivos submetidos a angioplastia coronária percutânea (ATC) off-label entre setembro de 2006 e dezembro de 2009. Comparamos os pacientes submetidos a ATC pelas vias radial e femoral em relação às evoluções hospitalar e tardia. Resultados: Predominaram na via radial pacientes mais jovens, do sexo masculino, com menor complexidade angiográfica, fato que se deveu à curva de aprendizado. Houve menor taxa de eventos cardíacos adversos maiores (ECAM), óbito e revascularização do vasoalvo na via radial, tanto na fase hospitalar como na fase tardia, em virtude do perfil clínico-angiográfico mais favorável. A via femoral foi preditor independente de ECAM hospitalar. A curva de sobrevivência ajustada, no entanto, mostrou que a via de acesso não teve influência significativa nos eventos clínicos a longo prazo. Conclusão: A técnica radial é segura na abordagem de pacientes selecionados com indicação off-label, apresentando resultados clínicos satisfatórios nas evoluções inicial e tardia.

DESCRITORES: Artéria radial. Angioplastia. Aprendizagem.

1 Hospital Beneficência Portuguesa de São Paulo - São Paulo, SP, Brasil. Correspondência: Clarissa Campo Dall'Orto. Rua Maestro Cardim, 769 - Bela Vista - São Paulo, SP, Brasil - CEP 01323-900 E-mail: clarissadallorto@ig.com.br

Recebido em: 20/2/2010 • Aceito em: 1\%/6/2010

\section{ABSTRACT}

\section{Coronary Angioplasty in Off-Label Indications: Comparison of Radial vs. Femoral Approach}

Background: There is increasing interest in the use of the radial approach in diagnostic and therapeutic procedures due to several advantages such as patient comfort in the immediate post-procedure with early return to daily routine activities, decreased hospitalization time and consequent reduction of hospital costs and low puncture site complication rates when compared with the femoral approach, reducing the rate of major bleeding, which is in turn related to increased risk of death and ischemic events. Method: Retrospective analysis of 1,807 consecutive patients undergoing off-label percutaneous transluminal coronary angioplasty (PTCA) from September 2006 to December 2009. The outcome of patients undergoing PTCA using the radial and femoral approaches during hospitalization and late follow-up were compared. Results: The radial approach prevailed in younger, male patients with lower angiographic complexity, which was due to the learning curve. Major adverse cardiac events (MACE), death and target-vessel revascularization rates were lower when the radial approach was used, both during hospitalization and in the late follow-up due to a more favorable clinical-angiographic profile. The femoral approach was an independent predictor of hospital MACE. The adjusted survival curve, however, showed that the access route did not have a significant impact on long-term clinical events. Conclusion: The transradial approach is safe when used in selected patients with off-label indication, providing good clinical results in the early and late follow-up.

KEY-WORDS: Radial artery. Angioplasty. Learning.
A utilização da artéria radial como via de acesso para procedimentos terapêuticos iniciou-se com Kimeneij, Laarman e Melker, em 19921,2, e desde então é objeto de crescente interesse para procedimentos diagnósticos e terapêuticos, em todo o mundo e também em nosso meio, por possuir diversas vantagens, como comodidade para o paciente no pós-procedimento imediato, retorno precoce a suas atividades, diminuição do tempo de internação com conse- 
quente redução dos custos hospitalares, e baixo índice de complicação do sítio de punção, reduzindo a taxa de sangramento maior, que, por sua vez, está relacionada, em muitos estudos, ao aumento do risco de morte e eventos isquêmicos. ${ }^{3-5}$

Apesar dessas vantagens, a maioria dos centros apresenta experiência limitada com a via radial, pois esta, sabidamente, requer curva de aprendizado mais longa que a via femoral, utilizada na maioria dos casos para procedimentos tanto diagnósticos como terapêuticos.

A escolha do local de acesso vascular na maioria dos centros é mais uma questão de tradição, opinião e experiência do operador que uma decisão baseada em evidência.

O objetivo deste estudo foi comparar o perfil clínico-angiográfico, a taxa de sucesso, e os desfechos hospitalares e tardios dos pacientes com indicação de angioplastia coronária off-label tratados pelas vias radial e femoral.

\section{MÉTODO}

Avaliamos, de forma retrospectiva, os resultados de pacientes submetidos a angioplastia coronária pelas vias radial ou femoral, de setembro de 2006 a dezembro de 2009. Nesse período foram realizadas 1.863 angioplastias coronárias off-label, utilizando stents convencionais ou farmacológicos, sendo $580(31,1 \%)$ realizadas por via radial, com 741 lesões tratadas, e 1.283 $(68,9 \%)$ por via femoral, com 1.676 lesões tratadas.

Foram incluídos pacientes com lesões localizadas em vasos $<2,5 \mathrm{~mm}$ ou $>3,5 \mathrm{~mm}$, com extensão $>20 \mathrm{~mm}$, localizadas em óstio, bifurcações, tronco de coronária esquerda ou enxertos arteriais ou venosos, lesões reestenóticas, oclusões crônicas ( $>3$ meses), pacientes com infarto agudo do miocárdio $(<48$ horas de evolução) e aqueles com fração de ejeção do ventrículo esquerdo $<30 \%$. ${ }^{6}$ Foram excluídos somente pacientes que apresentassem contraindicação para o uso da terapêutica dupla antiagregante plaquetária.

A seleção do paciente para a via radial ou para a via femoral era realizada a critério do operador, levando-se em consideração sua experiência, condições vasculares locais e preferência do paciente.

A punção da artéria radial foi realizada com a paciente com o membro superior direito estendido ao lado do corpo, com a palma da mão virada para cima, com anestesia local realizada com $2 \mathrm{ml}$ a $3 \mathrm{ml}$ de lidocaína 2\%. Utilizamos para puncionar jelco 18 ou 20, introdutores de fino calibre e paredes delgadas, que estão associados a menor formação de hematoma após uma primeira tentativa sem sucesso. Após a punção, utilizamos bainhas arteriais específicas para a artéria radial, $5 \mathrm{~F}$ a $7 \mathrm{~F}$, e administramos $10 \mathrm{mg}$ de mononitrato de isossorbida intra-arterial. Após o procedimento, era retirada a bainha arterial em sala, sem reversão da heparina, e feito curativo compressivo com gaze e esparadrapo, mantido habitualmente por duas a três horas após o procedimento.

Na via femoral, a punção era realizada após anestesia local com $15 \mathrm{ml}$ a $20 \mathrm{ml}$ de lidocaína $2 \%$, pela técnica de Seldinger, com agulha de punção 18 gauge, e introduzidas bainhas arteriais com diâmetro de $5 \mathrm{~F}$ a 7 F. Após o procedimento, a bainha arterial era retirada, o local da punção era comprimido manualmente por 20 minutos, e era feito curativo compressivo também com gaze e esparadrapo. O paciente era mantido em repouso com o membro estendido, durante três a cinco horas, conforme o calibre da bainha arterial.

Nas duas técnicas, a heparinização seguia-se de maneira usual, como indicado para angioplastia coronária, na dose de $70 \mathrm{UI} / \mathrm{kg}$ a $100 \mathrm{UI} / \mathrm{kg}$ por via intracoronária. Todos os pacientes receberam ácido acetilsalicílico e clopidogrel conforme as recomendações das diretrizes vigentes.

O acompanhamento clínico foi realizado por consulta médica ou por contato telefônico aos 30 dias, seis meses e um ano após angioplastia coronária. A partir de então, os pacientes eram avaliados anualmente.

O desfecho primário do estudo foi a taxa de eventos cardíacos adversos maiores na fase hospitalar. Os desfechos secundários incluíram: sucesso do procedimento, complicações vasculares e eventos cardíacos adversos maiores no seguimento tardio.

Eventos cardíacos adversos maiores intra-hospitalares foram definidos como óbito, infarto agudo do miocárdio e revascularização de urgência do vaso-alvo. Evento cardíaco adverso maior tardio foi definido como morte, infarto agudo do miocárdio e necessidade de nova revascularização do vaso-alvo, cirúrgica ou percutânea. Caracterizou-se infarto agudo do miocárdio quando ocorreu o surgimento de nova onda $Q$ em duas ou mais derivações contíguas ou elevação da creatina quinase fração $\mathrm{MB}$ (CK-MB) maior que três vezes o limite superior da normalidade. Sucesso angiográfico foi atingido quando se obteve lesão residual $<20 \%$ na presença de fluxo TIMI 3. O sucesso do procedimento foi definido como sucesso angiográfico na ausência de complicações maiores (óbito, infarto agudo do miocárdio ou necessidade de nova revascularização urgente). ${ }^{7}$ As complicações vasculares incluíram: pseudoaneurisma, hematoma, fístula arteriovenosa, sangramento externo, hematoma retroperitoneal, trombose arterial, abscesso do local de punção e aneurisma micótico. ${ }^{8}$

\section{Análise estatística}

Variáveis demográficas, clínicas, angiográficas e do procedimento foram comparadas entre os grupos pelo teste do qui-quadrado de Pearson ou exato de Fisher, quando apropriado. A idade foi comparada pelo teste de Mann-Whitney, pois a normalidade dos dados foi rejeitada em pelo menos um dos grupos. 
Dall'Orto CC, et al. Angioplastia Coronária nas Indicações Off-Label: Comparação das Vias Radial vs. Femoral. Rev Bras Cardiol Invasiva. 2010;18(2):177-84.

Regressão logística foi realizada para avaliar o impacto das características dos pacientes no evento cardíaco adverso maior hospitalar. Odds ratio (OR) e seus respectivos intervalos de confiança (IC 95\%) foram apresentados para quantificar os efeitos.

Para avaliação dos eventos cardíacos adversos maiores de longo prazo, a análise da sobrevivência livre do evento foi realizada pelo método de Kaplan-Meier, com comparação entre os grupos feita pelo teste de Log-Rank. O modelo de regressão de Cox foi ajustado para verificação de efeitos das demais variáveis clinicamente relevantes nos eventos cardíacos adversos maiores de longo prazo.

Valor de $\mathrm{P}<0,05$ foi considerado significante. Foi utilizado o programa SPSS for Windows versão 12.0 para a execução dos cálculos estatísticos.

\section{RESULTADOS}

Com relação às características clínicas e demográficas (Tabela 1), o grupo via radial apresentou indivíduos mais jovens, com menor porcentual de pacientes do sexo feminino e cirurgia de revascularização do miocárdio prévia. O espectro de apresentação clínica também foi diferente entre as vias radial e femoral.

$\mathrm{Na}$ análise das características angiográficas e do procedimento (Tabela 2), houve diferença no número de vasos acometidos. A necessidade de realização da troca da via de acesso, por falha de punção, existiu somente na via radial. O uso do inibidor da glicoproteína Ilb/IIla não foi diferente entre os grupos. Stents farmacológicos foram utilizados em 215 pacientes (37\%) na via radial e em 313 pacientes (24\%) na via femoral $(\mathrm{P}<0,0001)$. Pode-se observar a distribuição das lesões off-label entre os grupos na Tabela 3. Os pacientes com lesões de bifurcação, enxertos venosos ou arteriais, tronco de coronária esquerda não-protegido e lesões ostiais foram mais frequentemente tratados pela via femoral.

Como pode ser observado na Tabela 4, no grupo via radial houve maior sucesso do procedimento $(96,2 \%$ vs $93,0 \% ; P=0,008)$, menor ocorrência de eventos cardíacos adversos maiores hospitalares (1,7\% vs. 3,7\%; $\mathrm{P}=0,024)$, óbito $(0,5 \%$ vs. $1,8 \% ; \mathrm{P}=0,03)$ e complicações vasculares $(0,5 \%$ vs. $2,5 \% ; P=0,01)$. Não houve diferença nas taxas de infarto agudo do miocárdio, na necessidade de revascularização urgente, no sangramento maior pelo critério TIMI ou na insuficiência renal aguda.

Quando aplicamos o modelo de regressão logística (Tabela 5), observamos que idade, tabagismo, lesão de tronco de coronária esquerda e utilização de inibidor da glicoproteína llb/Illa comportaram-se como preditores independentes de evento cardíaco adverso maior hospitalar. Observamos efeito marginalmente significante para diabetes melito e via femoral nesses resultados. As estimativas sugerem que a chance de eventos cardíacos adversos maiores na fase hospitalar foi 2,7 vezes maior nos tabagistas, 3,7 vezes maior nos pacientes com lesão de tronco de coronária esquerda, e 6,1 vezes maior nos que utilizaram inibidor da glicoproteína IIb/IIla, e que a cada aumento de um ano na idade aumentaram as chances de eventos cardíacos adversos maiores em 4,2\%.

TABELA 1

Características clínicas e demográficas

\begin{tabular}{|c|c|c|c|}
\hline & $\begin{array}{c}\text { VR } \\
(n=580)\end{array}$ & $\begin{array}{c}\text { VF } \\
(n=1.283)\end{array}$ & $\mathbf{P}$ \\
\hline Idade, anos & $64,6 \pm 11,5$ & $64,59 \pm 11,45$ & 0,015 \\
\hline Sexo feminino & $138(23,8 \%)$ & $436(34 \%)$ & $<0,0001$ \\
\hline Dislipidemia & $386(66,6 \%)$ & $847(66 \%)$ & 0,821 \\
\hline Hipertensão arterial & $448(77,2 \%)$ & $1.011(78,8 \%)$ & 0,450 \\
\hline Diabetes melito & $143(24,7 \%)$ & $352(27,4 \%)$ & 0,208 \\
\hline Tabagismo & $141(24,3 \%)$ & $242(18,9 \%)$ & 0,007 \\
\hline RM prévia & $60(10,3 \%)$ & $275(21,4 \%)$ & $<0,0001$ \\
\hline Quadro clínico & & & 0,001 \\
\hline Assintomático & $93(16 \%)$ & $158(12,3 \%)$ & \\
\hline Angina estável & $239(41,3 \%)$ & $605(47 \%)$ & \\
\hline SCASST & 207 (35,7\%) & $386(30,1 \%)$ & \\
\hline SCACST & $40(6,9 \%)$ & $127(9,9 \%)$ & \\
\hline
\end{tabular}

$\mathrm{n}$ = número de pacientes; $\mathrm{RM}$ = cirurgia de revascularização miocárdica; SCACST = síndrome coronária aguda com supradesnivelamento do segmento ST; SCASST = síndrome coronária aguda sem supradesnivelamento do segmento ST; VF = via femoral; $V R=$ via radial. 
TABELA 2

Características angiográficas e do procedimento

\begin{tabular}{|c|c|c|c|}
\hline & $\begin{array}{c}\text { VR } \\
(n=580)\end{array}$ & $\begin{array}{c}\text { VF } \\
(n=1.283)\end{array}$ & $\mathbf{P}$ \\
\hline Número de vasos acometidos & & & 0,002 \\
\hline Uniarteriais & $213(36,7 \%)$ & $415(32,3 \%)$ & \\
\hline Biarteriais & $209(36 \%)$ & $413(32,2 \%)$ & \\
\hline Triarteriais & $158(27,2 \%)$ & $455(35,5 \%)$ & \\
\hline Lesão de TCE & $7(1,2 \%)$ & $42(3,3 \%)$ & 0,01 \\
\hline Uso de inibidor da GP IIb/IIla & $24(4,1 \%)$ & $77(6,6 \%)$ & 0,125 \\
\hline Uso de SF & $215(37 \%)$ & $313(24 \%)$ & $<0,0001$ \\
\hline Crossover da via de acesso & $16(3 \%)$ & 0 & $<0,0001$ \\
\hline Sucesso angiográfico & $568(98 \%)$ & $1.240(96,7 \%)$ & 0,172 \\
\hline
\end{tabular}

TABELA 3

Distribuição das lesões off-label

\begin{tabular}{lccc}
\hline & $\begin{array}{c}\text { VR } \\
(\mathbf{n}=\mathbf{7 4 1})\end{array}$ & $\begin{array}{c}\text { VF } \\
(\mathbf{n = 1 . 6 7 6 )}\end{array}$ & $\mathbf{P}$ \\
\hline Disfunção grave de VE* & $25(4,3 \%)$ & $65(5 \%)$ & 0,557 \\
Reestenose intrastent & $37(5 \%)$ & $62(3,7 \%)$ & 0,171 \\
Lesão em bifurcação & $58(7,8 \%)$ & $209(12,5 \%)$ & 0,001 \\
PVS ou enxerto de mamária & $23(3,1 \%)$ & $192(11,5 \%)$ & $<0,0001$ \\
Comprimento > 20 mm & $315(42,5 \%)$ & $557(33,2 \%)$ & $<, 0001$ \\
Vasos $\leq 2,5$ mm ou $\geq 3,5 \mathrm{~mm}$ & $588(79,4 \%)$ & $1.284(76,6 \%)$ & 0,152 \\
Lesão de TCE não protegido & $1(0,1 \%)$ & $21(1,3 \%)$ & 0,015 \\
Oclusão total & $142(19,2 \%)$ & $308(18,4 \%)$ & 0,688 \\
Lesão ostial & $48(6,5 \%)$ & $193(11,5 \%)$ & $<0,0001$ \\
\hline
\end{tabular}

* Porcentual em relação ao número de pacientes $(\mathrm{VR}=580$ e $\mathrm{VF}=1.283)$.

$\mathrm{n}$ = número de pacientes; $\mathrm{PVS}=$ ponte de veia safena; $\mathrm{TCE}=$ tronco de coronária esquerda; $\mathrm{VE}=$ ventrículo esquerdo; $\mathrm{VF}=$ via femoral; $\mathrm{VR}=$ via radial.

No seguimento clínico (Tabela 6), 85,8\% dos pacientes foram acompanhados num período médio de $569 \pm 397$ dias. Os eventos cardíacos adversos maiores na fase tardia foram menores na via radial $(3,7 \%$ vs. $7,7 \% ; P<0,0001)$. A incidência de óbito $(0,5 \%$ vs. $3,4 \% ; \mathrm{P}<0,0001)$, a revascularização do vaso-alvo (1,8\% vs. 4,6\%; $P=0,001)$ ou a necessidade de nova revascularização (3,5\% vs. 6,5\%; $\mathrm{P}<0,0001)$ foram menores na via radial. Não houve diferença na taxa de trombose dos stents entre os grupos (Tabela 7).

Um modelo de regressão de Cox foi ajustado para avaliação do efeito das covariáveis no tempo de ocorrência do evento cardíaco adverso maior tardio (Tabela 8). Existem evidências de aumento de 73,3\% de risco da ocorrência de eventos cardíacos adversos maiores nos pacientes triarteriais $(P=0,03)$. Os quadros clínicos assintomáticos e angina estável mostraram- se fatores protetores, diminuindo a chance da ocorrência do evento cardíaco adverso maior tardio em 97,5\% e $74,4 \%$, respectivamente. O efeito da via de acesso não foi significativo na presença dos demais fatores.

A curva de sobrevivência livre de eventos cardíacos adversos maiores (Figura 1) é maior na via radial que na via femoral. Porém, analisando-se a Figura 2, que nos mostra a curva de sobrevivência ajustada, observa-se que as duas curvas tornam-se superpostas, pois a via de acesso perde seu efeito no evento cardíaco adverso maior tardio na presença das outras variáveis.

\section{DISCUSSÃO}

Em nosso registro, cerca de um terço dos pacientes com indicação de angioplastia coronária off-label foi abordado pela via radial. Os pacientes tratados por 
Dall'Orto CC, et al. Angioplastia Coronária nas Indicações Off-Label: Comparação das Vias Radial vs. Femoral. Rev Bras Cardiol Invasiva. 2010;18(2):177-84.

TABELA 4

Evolução hospitalar

\begin{tabular}{lccc}
\hline & $\begin{array}{c}\text { VR } \\
(\mathbf{n}=\mathbf{5 8 0})\end{array}$ & $\begin{array}{c}\text { VF } \\
(\mathbf{n = 1 . 2 8 3})\end{array}$ & $\mathbf{P}$ \\
\hline Sucesso clínico & $558(96,2 \%)$ & $1193(93 \%)$ & 0,008 \\
ECAM & $10(1,7 \%)$ & $47(3,7 \%)$ & 0,024 \\
Óbito & $3(0,5 \%)$ & $23(1,8 \%)$ & 0,03 \\
IAM & $4(0,7 \%)$ & $20(1,6 \%)$ & 0,123 \\
RVA urgente & $3(0,5 \%)$ & $4(0,3 \%)$ & 0,526 \\
Insuficiência renal aguda & $5(0,9 \%)$ & $12(0,9 \%)$ & 0,878 \\
Sangramento maior* & $1(0,2 \%)$ & $4(0,3 \%)$ & $>0,99$ \\
Complicações vasculares** & $3(0,5 \%)$ & $30(2,5 \%)$ & 0,01 \\
\hline
\end{tabular}

* Sangramento maior segundo critérios de TIMI.

** Pseudoaneurisma, hematoma, fístula arteriovenosa, sangramento externo, hematoma retroperitoneal, trombose arterial, abscesso do local de punção e aneurisma micótico.

ECAM = eventos cardíacos adversos maiores (óbito, infarto agudo do miocárdio ou revascularização do vaso-alvo urgente); IAM = infarto agudo do miocárdio; $\mathrm{n}=$ número de pacientes; $\mathrm{RVA}=$ revascularização do vaso-alvo; $\mathrm{VF}=$ via femoral; $\mathrm{VR}=\mathrm{via}$ radial.

TABELA 5

Resultados do modelo logístico para avaliação de ECAM hospitalar

\begin{tabular}{lccccc}
\hline & Efeito & $\begin{array}{c}\text { Erro } \\
\text { padrão }\end{array}$ & OR & IC 95\% & $\begin{array}{c}\text { Valor } \\
\text { de P }\end{array}$ \\
\hline Tabagismo & 0,979 & 0,323 & 2,661 & $1,413-5,013$ & 0,0025 \\
Diabetes melito & 0,544 & 0,294 & 1,722 & $0,968-3,062$ & 0,0642 \\
Lesão de TCE & 1,317 & 0,506 & 3,732 & $1,383-10,068$ & 0,0093 \\
Inibidor da GP IIb/IIla & 1,813 & 0,348 & 6,127 & $3,098-12,118$ & $<0,0001$ \\
Acesso femoral & 0,656 & 0,359 & 1,928 & $0,953-3,899$ & 0,0678 \\
Idade & 0,041 & 0,013 & 1,042 & $1,015-1,069$ & 0,0022 \\
Constante & $-7,357$ & 1,002 & 0,001 & & \\
\hline
\end{tabular}

Teste de Hosmer e Lemeshow $(P=0,591)$.

GP = glicoproteína; IC 95\% = intervalo de confiança de 95\%; OR = odds ratio; TCE = tronco de coronária esquerda.

TABELA 6

Evolução clínica tardia

\begin{tabular}{|c|c|c|c|}
\hline & VR & VF & $\mathbf{P}$ \\
\hline Pacientes acompanhados & $510(88 \%)$ & $1.088(84,8 \%)$ & 0,086 \\
\hline Quadro clínico & & & 0,005 \\
\hline Assintomático & $468(91,7 \%)$ & $923(84,9 \%)$ & \\
\hline Angina estável & $26(5 \%)$ & $102(9,4 \%)$ & \\
\hline SCASST & $6(1,2 \%)$ & $16(1,4 \%)$ & \\
\hline ECAM & $19(3,7 \%)$ & $97(7,7 \%)$ & $<0,0001$ \\
\hline Óbito & $3(0,5 \%)$ & $37(3,4 \%)$ & $<0,0001$ \\
\hline IAM & $7(1,3 \%)$ & $10(0,9 \%)$ & 0,583 \\
\hline RVA & $9(1,8 \%)$ & $50(4,6 \%)$ & 0,001 \\
\hline NNR & $18(3,5 \%)$ & $84(6,5 \%)$ & $<0,0001$ \\
\hline
\end{tabular}


TABELA 7

Taxa de trombose entre os grupos

\begin{tabular}{|c|c|c|c|c|c|}
\hline & \multicolumn{2}{|c|}{ VR } & \multicolumn{2}{|c|}{ VF } & \multirow[b]{2}{*}{$\mathbf{P}$} \\
\hline & SF & SC & SF & SC & \\
\hline Grau de certeza* & & & & & 0,213 \\
\hline Definitiva & $2(0,35 \%)$ & $2(0,35 \%)$ & 0 & $5(0,4 \%)$ & \\
\hline Possível & 0 & 0 & $4(0,31 \%)$ & $2(0,15 \%)$ & \\
\hline Provável & 0 & 0 & 0 & $3(0,3 \%)$ & \\
\hline Classificação temporal & & & & & 0,851 \\
\hline Aguda & 0 & 0 & 0 & 0 & \\
\hline Subaguda & $2(0,35 \%)$ & 0 & 0 & $6(0,5 \%)$ & \\
\hline Tardia & 0 & $2(0,35 \%)$ & $4(0,35 \%)$ & $4(0,35 \%)$ & \\
\hline Muito tardia & 0 & 0 & 0 & 0 & \\
\hline
\end{tabular}

TABELA 8

Resultados do modelo de Cox para avaliação de evento cardiovascular adverso maior tardio

\begin{tabular}{lccccc}
\hline & Efeito & $\begin{array}{c}\text { Erro } \\
\text { padrão }\end{array}$ & OR & IC 95\% & P \\
\hline Extensão DAC biarterial & 0,482 & 0,263 & 1,619 & $0,966-2,712$ & 0,0674 \\
Extensão DAC triarterial & 0,550 & 0,256 & 1,733 & $1,049-2,864$ & 0,0319 \\
Assintomático & $-3,676$ & 0,239 & 0,025 & $0,016-0,04$ & $<0,0001$ \\
Angina estável & $-1,363$ & 0,239 & 0,256 & $0,16-0,409$ & $<0,0001$ \\
SCASST & $-0,509$ & 0,410 & 0,601 & $0,269-1,343$ & 0,2148 \\
\hline
\end{tabular}

Extensão DAC uniarterial: categoria de referência.

DAC = doença arterial coronária; IC 95\% = intervalo de confiança de 95\%; OR = odds ratio; SCASST = síndrome coronária aguda sem supradesnivelamento do segmento ST.

essa via mostraram menor complexidade clínica e angiográfica e melhor evolução clínica inicial e tardia. A análise de regressão logística identificou maior risco de eventos clínicos hospitalares pela via de acesso femoral. A curva de sobrevivência ajustada, no entanto, mostrou que a via de acesso não teve influência significativa nos eventos clínicos tardios.

A menor complexidade clínica e angiográfica da via radial pode ser explicada pelo fato de pacientes com cirurgia de revascularização do miocárdio prévia, lesões em bifurcação e lesões de tronco de coronária esquerda não-protegido terem sido abordados preferencialmente pela via femoral durante a curva de aprendizado da nova técnica. O sexo feminino também foi mais frequentemente abordado pela via femoral, pois apresenta artérias mais finas e com maior chance de espasmo, fato que dificulta o procedimento na fase inicial do treinamento. Como consequência, observamos maior sucesso e menor incidência de óbito, revascularização do vaso-alvo e eventos cardíacos adver- sos maiores na via radial A utilização mais frequente dos stents farmacológicos na via radial também certamente influenciou esses resultados. Após o ajuste das variáveis no modelo de Cox, apenas a extensão da doença arterial coronária manteve-se como fator preditor independente da ocorrência de eventos cardíacos adversos maiores. Esse resultado diferiu da literatura, em que os pacientes da via femoral cursam com maiores taxas de eventos cardíacos adversos maiores relacionados ao sangramento maior ${ }^{9}$ e não pela maior complexidade clínica e angiográfica.

A via radial, na nossa experiência, mostrou-se uma técnica segura e eficaz, com alto sucesso angiográfico em angioplastias coronárias off-label selecionadas, dentre elas as lesões de enxertos venosos e também arteriais, incluindo enxertos de artéria mamária interna esquerda, nos quais utilizamos a via radial esquerda. A via radial também pode ser utilizada com sucesso no tratamento da bifurcação, pois comporta com facilidade cateteres $6 \mathrm{~F}$, possibilitando assim a utilização de dois 


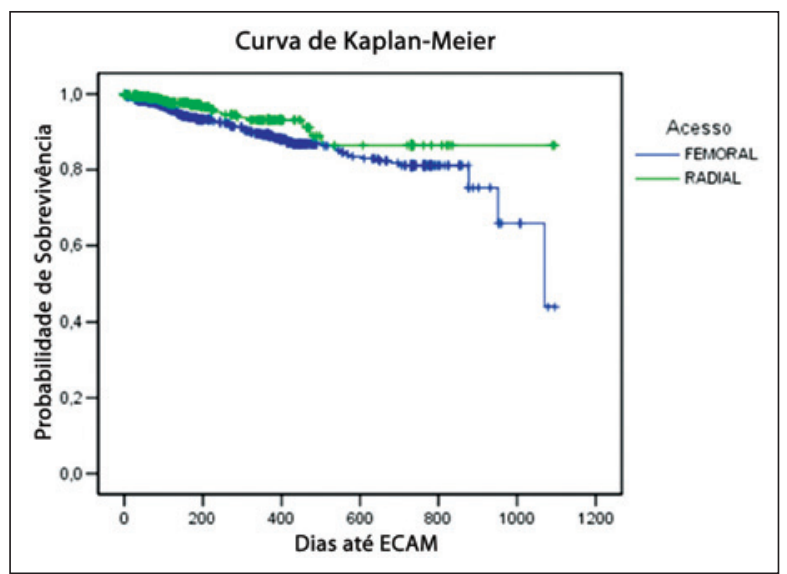

Figura 1 - Sobrevivência livre de eventos cardíacos adversos maiores. ECAM = eventos cardíacos adversos maiores.

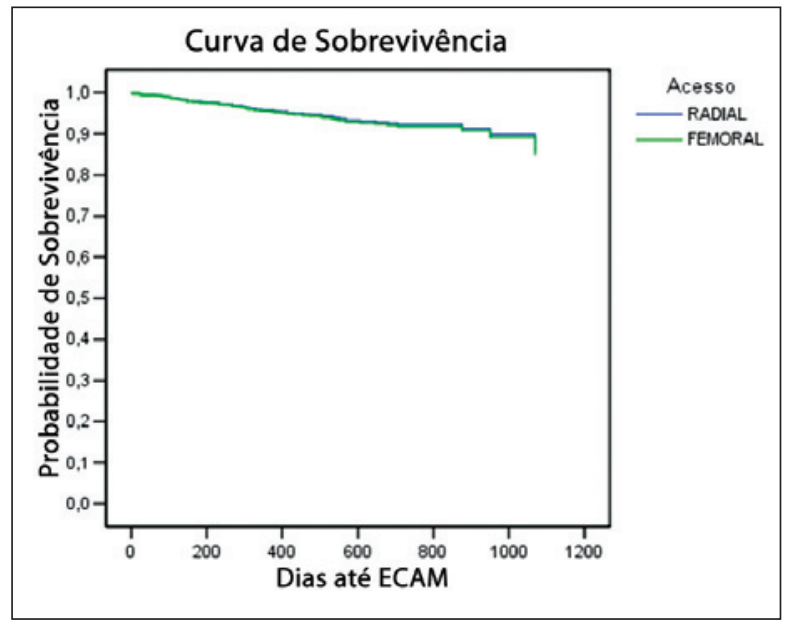

Figura 2 - Curva de sobrevivência livre de eventos cardíacos adversos maiores ajustada

balões simultaneamente. Por outro lado, essa prática não é possível com dois stents concomitantemente. Acreditamos que para o tratamento bem-sucedido das lesões de bifurcação por via radial necessitamos de um planejamento criterioso.

A via radial possui a vantagem de apresentar menor taxa de complicações vasculares, como demonstrado pelo estudo ACCESS $^{10}$, em que foi comparada com a braquial e a femoral, e de diminuir, assim, a taxa de sangramentos maiores, eventos isquêmicos e óbito. . $^{3,11}$

Labrunie et al. ${ }^{9}$, em estudo recente, também demonstraram a eficácia da via radial quando comparada às vias clássicas (Sones e via femoral) na realização de coronariografia, com índice de sucesso e complicações similares.

Também há consenso na literatura quanto à necessidade da fase de aprendizado, fato demonstrado por inúmeros trabalhos. ${ }^{12-14}$
Além de apresentar menor taxa de complicações vasculares, tornando-se alternativa em obesos, pacientes com doença aortoilíaca, em tratamento com anticoagulante, inibidores da glicoproteína Ilb/IIla ou na fase aguda do infarto tratado com trombolíticos, ainda podemos ressaltar inúmeras vantagens da técnica radial: 1) não há grandes nervos ou veias adjacentes à artéria radial, tornando muito baixo o risco de lesão nervosa ou fístula arteriovenosa ${ }^{15,16}$; 2) maior conforto para o paciente, pois essa técnica permite a deambulação imediata, resultando em maior satisfação do paciente, fato já demonstrado em estudos randomizados; 3) redução da intensidade e do tempo gasto nos cuidados de enfermagem com a via de acesso; e 4) alta hospitalar precoce com redução de custos.

\section{LIMITAÇÕES DO ESTUDO}

Por se tratar de um estudo observacional, reconhecemos as limitações que são inerentes a esse tipo de estudo. Não houve randomização dos pacientes que seriam selecionados para abordagem radial e femoral, e isso foi decidido pelo operador, avaliando-se as condições vasculares locais e levando-se em consideração a complexidade das lesões coronárias.

As diversas diferenças entre os grupos não podem ser ajustadas totalmente pela análise multivariada realizada, em virtude do pequeno número de variáveis independentes incluídas, quando os preditores de eventos nessa população incluem muitas outras características.

Não calculamos o tempo de fluoroscopia, que é um medidor da complexidade do procedimento, e que, na literatura, normalmente é maior que na via radial.8,14 Também não foram avaliados o tempo de permanência e o custos na fase hospitalar.

\section{CONCLUSÃO}

A técnica radial é segura na abordagem de pacientes selecionados com lesões off-label, demonstrando resultados clínicos satisfatórios nas evoluções inicial e tardia. É necessário que a proficiência na técnica seja alcançada, pois a via radial é uma abordagem mais desafiadora e que demanda curva de aprendizado mais prolongada, comparativamente à via femoral.

\section{CONFLITO DE INTERESSES}

Os autores declararam inexistência de conflito de interesses relacionado a este manuscrito.

\section{REFERÊNCIAS}

1. Kiemeneij F, Laarman GJ, Melker E. Transradial artery coronary angiography. Am Heart J. 1995;129:1-7.

2. Kiemeneij F, Laarman GJ, Melker E. Transradial artery PalmazSchatz coronary stent implantation: results of a single center feasibility study. Am Heart J. 1995;130:14-21.

3. Eikelboom JW, Mehta SR, Anand SS, Xie C, Fox KAA, Yusuf 
S. Adverse impact of bleeding on prognosis in patients with acute syndromes. Circulation. 2006;114:774-82.

4. Rao SV, O'Grady K, Pieper KS, Granger CB, Newby KL, Werf $\mathrm{FV}$, et al. Impact of bleeding severity on clinical outcomes among patients with acute coronary syndromes. Am J Cardiol. 2005;96:1200-6.

5. Jolly SS, Amlani S, Hamon M, Yusuf S, Mehta SR. Radial versus femoral access for coronary angiography or intervention and impact on major bleeding and ischemic events: a systematic review and meta-analysis or randomized trials. Am Heart J. 2009;157(1):132-40.

6. Qasim A, Cosgrave J, Latib A, Colombo A. Long-term followup of drug eluting stents inserted on- and off-label indications. Am J Cardiol. 2007;100:1619-24.

7. Smith SC Jr, Feldman TE, Hirshfeld JW Jr, Jacobs AK, Kern MJ, King SB $3^{\text {rd }}$, et al. ACC/AHA/SCAI 2005 Guideline Update for Percutaneous Coronary Intervention: a report of the American College of Cardiology/American Heart Association Task Force on Practice Guidelines. Circulation. 2006;113(7): e166-286.

8. Lumsden $\mathrm{AB}$, Peden EK, Bush RL, Lin PH. Complications of endovascular procedures. In: Rutherford RB, editor. Rutherford vascular surgery. $6^{\text {th }}$ ed. New York: Elsevier; 2005. p. 810.

9. Labrunie A, Tebet MA, Andrade PB, Andrade MVA, Conterno LO, Mattos LA, et al. Coronariografia via transradial: curva de aprendizagem, avaliada por estudo multicêntrico. Rev Bras Cardiol Invasiva. 2009;17(1):82-7.
10. Kiemeneij F, Laarman GJ, Odeekerken D, Slagboom T, van der Wieken R. A randomized comparison of percutaneous transluminal coronary angioplasty by the radial, brachial and femoral approaches: the Access Study. J Am Coll Cardiol. 1997;29(6):1269-75.

11. Doyle BJ, Rihal CS, Gastineau DA, Holmes DR Jr. Bleeding, blood transfusion, and increased mortality after percutaneous coronary intervention: implications for contemporary practice. J Am Coll Cardiol. 2009;53(22):2019-27.

12. Cooper CJ, El-Shiekh RA, Cohen DJ, Blaesing L, Burket MW, Basu A, et al. Effect of transradial access on quality of life and cost of cardiac catheterization: a randomized comparison. Am Heart J. 1999;138 (3 Pt 1):430-6.

13. Spaulding $C$, Lefèvre $T$, Funck $F$, Thébault $B$, Chaveau $M$, Ben Hamda K, et al. Left radial approach for coronary angiography: results of a prospective study. Cathet Cardiovasc Diagn. 1996;39(4):365-70.

14. Ludman PF, Stephens NG, Harcombe A, Lowe MD, Shapiro LM, Schofield PM, et al. Radial versus femoral approach for diagnostic coronary angiography in stable angina pectoris. Am J Cardiol. 1997;79(9):1239-41.

15. Valsecchi O, Vassileva A, Musumeci G, Rossini R, Tespili M, Guagliumi G, et al. Failure of transradial approach during coronary interventions: anatomic considerations. Catheter Cardiovasc Interv. 2006;67(6):870-8.

16. Rihal CS, Holmes DR Jr. Transradial cardiac catheterization: is femoral acess obsolete? Am Heart J. 1999;138(3 Pt 1):392-3. 